\title{
Public Health: An Emerging Specialty Among Medical Students in Francophone Sub-Saharan African Countries
}

\author{
Didier Koumavi Ekouevi ${ }^{1,2,3, \text { * }}$, Boris Tchounga ${ }^{3,4}$, Patrick Ahuatchi Coffie ${ }^{4,5,6}$, \\ Fifonsi Adjidossi Gbeasor-Komlanvi ${ }^{1,7}$, Mohamed Cissé ${ }^{8}$, Serge Paul Eholie ${ }^{5,6}$ \\ ${ }^{1}$ Public Health Department, University of Lomé, Lomé, Togo \\ ${ }^{2}$ Bordeaux School of Public Health (ISPED), University of Bordeaux, Bordeaux, France \\ ${ }^{3}$ Inserm, University of Bordeaux, Bordeaux, France \\ ${ }^{4}$ PACCI Research Center, Abidjan, Ivory Coast \\ ${ }^{5}$ Dermatology and Infectiology Department, University of Félix Houphouët Boigny, Abidjan, Ivory Coast \\ ${ }^{6}$ Treichville Teaching Hospital, Tropical and Infectious Diseases Unit, Abidjan, Ivory Coast \\ ${ }^{7}$ African Center for Research on Public Health and Epidemiology, Lomé, Togo \\ ${ }^{8}$ Dermatology Department, University Gamal Abdel Nasser, Conakry, Guinea
}

Email address:

Didier.ekouevi@gmail.com (D. K. Ekouevi)

${ }^{*}$ Corresponding author

\section{To cite this article:}

Didier Koumavi Ekouevi, Boris Tchounga, Patrick Ahuatchi Coffie, Fifonsi Adjidossi Gbeasor-Komlanvi, Mohamed Cissé, Serge Paul Eholie. Public Health: An Emerging Specialty Among Medical Students in Francophone Sub-Saharan African Countries. Central African Journal of Public Health. Vol. 5, No. 3, 2019, pp. 129-135. doi: 10.11648/j.cajph.20190503.15

Received: March 18, 2019; Accepted: April 26, 2019; Published: May 20, 2019

\begin{abstract}
Sub-Saharan African (SSA) countries severely lack financial and human resources while bearing the heaviest burden of disease. Provision of a sufficient and competent public health workforce is a key component of health system strengthening, but only few data are available on the specialty that health professionals and medical students of francophone SSA countries wish to pursue. The objective of the study was to assess future specialization preference of medical students and the ranking of public health by country in francophone countries. A web-based cross-sectional survey was conducted from April to July 2017 in medical schools of francophone SSA universities. All students in their last three years of medical training were eligible for the study and were approached via the internet platform of a West African network of medical students named "Réseau des Etudiants en Médecine de l'Afrique de l'Ouest" (REMAO), and the intranet of each university when available. A total of 1,161 medical students in francophone SSA countries were included. Public health was ranked first among medical specialties in francophone SSA countries, and it was chosen by $13.4 \%$ of medical students. The main motivations for this choice were the fact that they like the specialty $(52.4 \%)$, job salary and benefits $(39.9 \%)$, the fact that this specialty is fulfilling or matches with their personality $(31.3 \%)$, and the opportunity for conducting research $(23.1 \%)$. This is the first study on the choice of medical specialties among medical students in francophone SSA countries but also one of the rare studies reporting public health as medical students' preferred choice. Responding to this growing and urgent demand is critical not only to meet students' educational needs but also to improve national health systems with special focus on preventive medicine and governance.
\end{abstract}

Keywords: Choice, Medical Specialty, Medical Education and Training, Public Health Policy, Africa

\section{Introduction}

During the past five years, sub-Saharan African (SSA) countries have faced many health emergencies, such as
Dengue fever in Burkina Faso, yellow fever in Congo, and Ebola outbreak in West Africa [1-5]. Moreover, SSA region bears over $74 \%$ of the global burden of communicable diseases and $17 \%$ of the global burden of non-communicable diseases (NCDs) [6, 7]. Despite the adoption and 
implementation of effective health programs such as the expanded program on immunization, malaria prevention program, cancer screening program and other NCD prevention programs, the coverage of SSA's population health needs is still very low, especially in regards to preventable diseases [8-10]. This situation reflects the weakness of the health systems of SSA countries which sorely lack financial and human resources [11-13]. In fact, while bearing over $24 \%$ of the global burden of disease, SSA is home to only $3 \%$ of the global health workforce and accounts for only $1 \%$ of global health expenditures $[6,14]$.

To strengthen health systems of SSA countries, there is a critical need for medical specialists covering various health fields, including public health specialists who can address specific bottlenecks of these health systems [7, 11, 15]. In SSA, the average workforce density was around 0.8 health care workers (HCWs) per 1,000 people in 2006, while it was estimated that a minimum workforce density of $2.5 \mathrm{HCWs}$ per 1,000 people was necessary to achieve the millennium development goals (MDGs) [6]. More recently, the World Health Organization (WHO) estimated that to achieve $80 \%$ of sustainable development goals (SDGs), a minimum density of 4.45 skilled health workers (doctors, nurses, midwives) per 1,000 people was necessary, while the 2013 density of skilled health workers in SSA was estimated at only 2.2 per 1000 people [12]. Moreover, in the SSA mapping of the public health training capacities published in 2007, it was reported that only 500 full-time public health specialists were available for more than 900 million people, and that the shortage of both training programs and public health specialists was more profound in francophone SSA countries [16].

During the last decade, many initiatives targeting the development of public health workforce in SSA have emerged with the support of the WHO and local SSA organizations [11, 17-19]. Many training programs are now available across SSA including in francophone countries [2026]. However, few data are available on the willingness of health professionals and medical students of francophone SSA countries to engage in a public health training program. The objective of the study was to assess future specialization preference of medical students and the ranking of public health by country in francophone countries.

\section{Methods}

\subsection{Study Design and Settings}

A web-based cross-sectional survey was conducted from April to July 2017 in medical schools of francophone SSA universities. All students in their last three years of medical training were eligible for the study and were approached via the internet platform of a West African network of medical students named "Réseau des Etudiants en Médecine de l'Afrique de l'Ouest" (REMAO), and the intranet of each university when available. The link to the survey and an explanatory message were first sent to REMAO's delegates who subsequently shared them with all eligible students via emails, and social network messaging.

\subsection{Participants}

Medical students in francophone SSA countries usually spend seven or eight years to complete the medical doctor (MD) degree. In most francophone SSA countries, medical doctors who wish to specialize choose their preferred specialty and have to pass an exam before enrollment in the program. A limited number of places is generally available for each existing specialty by country.

\subsection{Measurements}

A 19-item web-questionnaire was developed on Survey Monkey to assess participants' demographic characteristics, their desire to specialize, their preferred choice for future specialization, the reasons supporting their choice and their preferred destination to specialize.

\subsection{Statistical Analysis}

Quantitative variables (age) were presented as median with interquartile range (IQR) and categorical variables (sex, year of medical school, future specialization preference) were presented as percentages. Pearson chi-square test was used to assess statistical significance of differences in the choice patterns of participants. Statistical significance was set at $p$ $<0.05$. Data analyses were performed using Stata software (Stata $^{\mathrm{TM}}$ 11.0 College Station, Texas, USA).

Ethical approval for this study was waived by the 'Comite de Bioéthique pour la Recherche en Santé du Ministère de la santé du Togo' (ethics committee from the Ministry of Health of Togo), because participants were thought to be at no risk of harm and there was no intervention.

\section{Results}

A total of 1,161 medical students living in SSA and studying medicine in 15 francophone SSA countries participated in the study. Participants were mainly students in West Africa (87.4\%), 727 (62.6\%) were male, 1,050 (90.5\%) were single and the median age was 25 years (IQR [23-27]). Among participants, $592(51.0 \%)$ were in their final year of medical school.

The willingness to choose a medical specialty was expressed by 1,150 (99.1\%) participants, of which 1,097 (95.4\%) had a clear idea on their choice. The top five medical specialties chosen by the participants were public health (13.4\%), cardiology $(10.7 \%)$, obstetrics and gynecology $(10.8 \%)$, pediatrics $(8.4 \%)$ and general surgery $(8.2 \%)$. Of the 15 participating countries, public health ranked first in three countries (Burkina Faso, Guinea-Conakry, and Togo), second in three countries (Congo, Mali and Niger), and third in one country (Côte d'Ivoire). In four countries (Burundi, Central African Republic, Chad and Rwanda), no student had chosen public health as a medical specialty. Among the 11 countries in which public health was chosen as future choice 
for specialization, the proportion of medical students choosing public health as medical specialty ranged from $4.8 \%$ in Senegal to $24.8 \%$ in Guinea-Conakry (figure 1). The choice of public health as a medical specialty was higher in men than in women $(17.0 \%$ vs. $7.3 \%, \mathrm{p}<0.001)$.

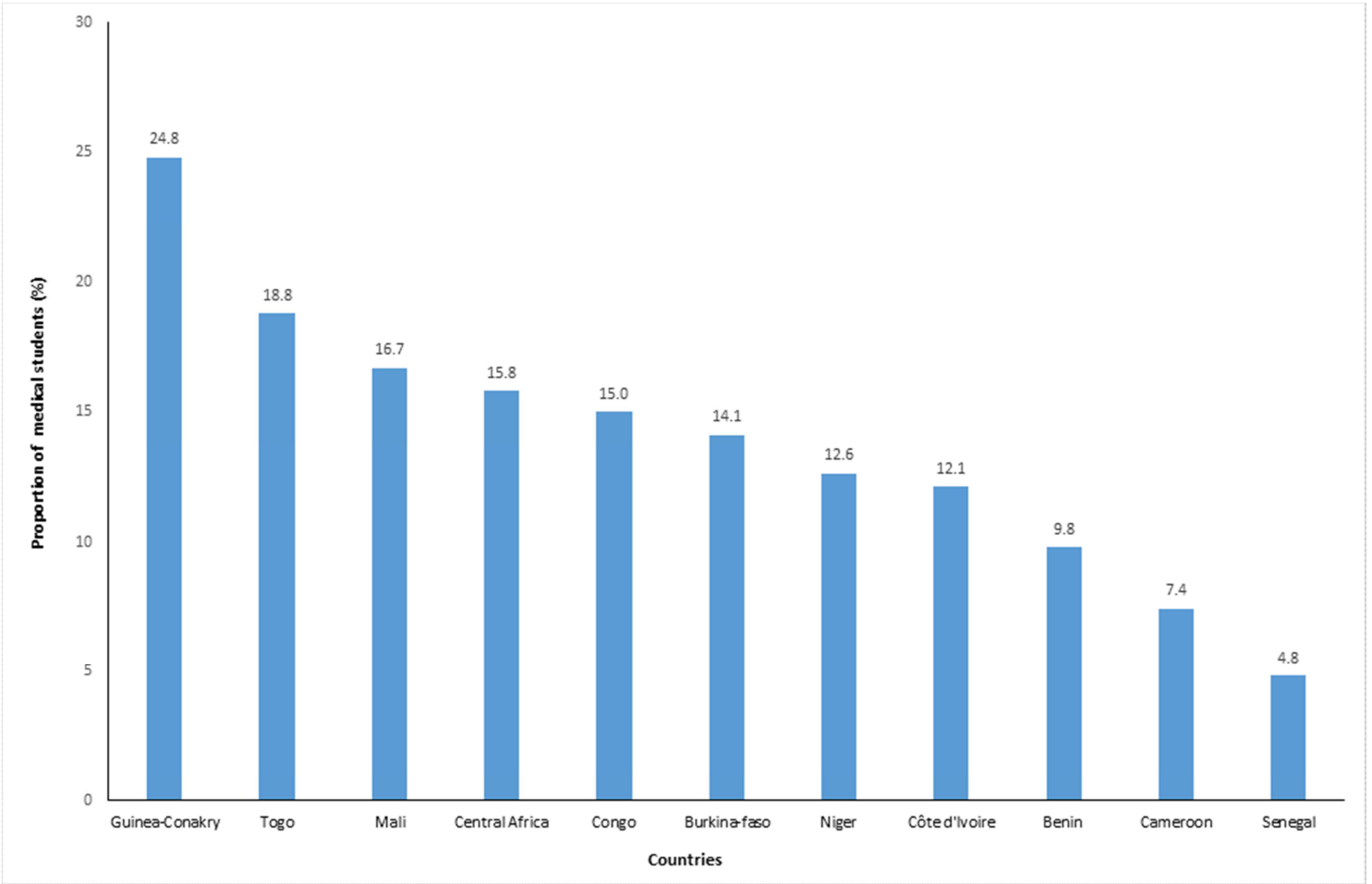

Figure 1. Choice of Public Health as medical specialty in francophone sub-Saharan Africa countries in 2017.

Foot note: Data are shown as proportions of medical students who chose Public Health by participating country. In four countries nobody has chosen public health.

In addition to loving the specialty $(52.4 \%)$, the main reasons for the choice of public health as specialty were the belief that this specialty is fulfilling or matches with the personality of the participant $(31.3 \%)$, the salary and benefits (39.9\%), the opportunity for conducting research $(23.1 \%)$, and the burden of an epidemic in participant's country or region (14.9\%) (figure 2$)$.

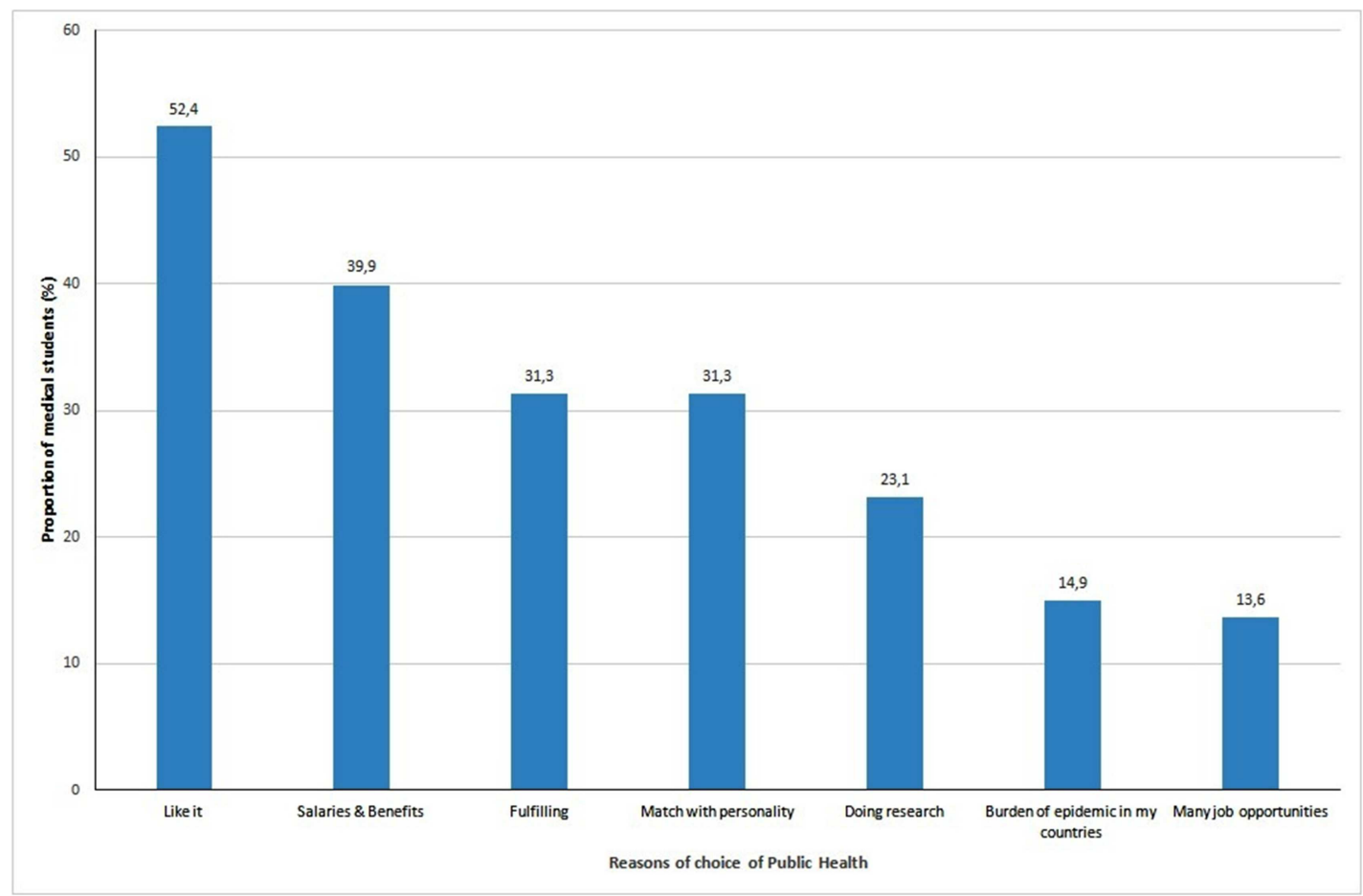

Figure 2. Reasons for choosing Public Health as preferred medical specialty in francophone sub-Saharan countries in 2017. 
Foot note: Data are shown as proportions of medical students for each selected motivating reason.

Among participants who wished to engage in public health as medical specialty, 19 (12.9\%) were planning on training for public health in their country of residence, $85(57.8 \%)$ were planning to travel abroad and the remaining 43 (29.3\%) did not make any choice. Among the 19 students who were planning to study public health in their country of residence, two third were concentrated in Senegal $(n=6)$ and Côte d'Ivoire $(n=5)$. Those who wished to specialize abroad identified Europe (44.4\%), North America (33.3\%) and others SSA countries $(22.3 \%)$ as preferred destinations. To support the costs of their specialty, most of these students indicated they would rely on personal resources $(30.1 \%)$, on their family (20.1\%), and on scholarships (27.9\%).

\section{Discussion}

Public health was ranked first among medical specialties in francophone SSA countries, and it was chosen by $13.4 \%$ of medical students. The main motivations for this choice were the fact that they like the specialty $(52.4 \%)$, job salary and benefits $(39.9 \%)$, the fact that this specialty is fulfilling or matches with their personality $(31.3 \%)$, and the opportunity for conducting research $(23.1 \%)$.

This is the first study on the choice of medical specialties among medical students in francophone SSA countries but also the first reporting public health as a preferred choice. This result is somewhat surprising, since similar pattern has rarely been reported in the literature. In studies previously conducted in Nigeria, Kenya and in other parts of the world, the top five listed medical specialties were surgery, pediatrics, obstetrics-gynecology, internal medicine and community medicine [27-32]. Only one survey conducted in Malawi reported public health as the preferred medical specialty for $28 \%$ of medical graduates from Blantyre College of medicine [33].

These findings in francophone SSA countries may reflect the increasing interest for public health among medical students. However, the situation may not be comparable in the different countries, since the choice of medical specialty also depends on the national policy for human resources in health, and the availability of training programs $[18,19,34$, 35]. In Guinea-Conakry where the choice of public health was the highest $(24.8 \%)$, this could be explained by the Ebola outbreak which stroke the country in 2014-2016 and which was one of the deadliest outbreak in SSA. During this epidemic, there was a crucial need for public health specialists to implement and manage response programs and activities [2, 36]. To overcome the human resource crisis during the Ebola outbreak in this country, the West African Health Organization (WAHO) organized short training programs for volunteering doctors from other West African countries who were subsequently deployed in the most affected countries. In addition, many Non-Governmental Organizations (NGOs) deployed public health specialists from Europe and North America with considerable financial and logistic resources to tackle weaknesses of local health systems. The success of the response to the Ebola outbreak, in terms of public health professionals' involvement and number of lives saved, probably gave more visibility to this discipline. Finally, the combination of the needs for qualified public health specialists and the perception of having a better position or opportunities as it is the case in other countries may have had an impact on the choice of this specialty.

Aside from Guinea-Conakry, the choice of public health as preferred medical specialty probably highlights the fact that public health is the discipline of the future, since it can help improve health systems' reactivity and more generally, populations' health. Health systems in several West African countries are essentially focused on curative activities in a context of a great shortage of skilled health workers [11, 34, 35]. With the rise of questions on access to universal health coverage and the growing burden of NCDs, there is an increased awareness regarding the lack of public health professionals with core competencies in health policy and management, health economics, and preventive medicine, to name a few. Choosing public health could have also been driven by the myth that public health specialists can easily join the United Nations System, with better position and comfortable salaries.

Thus, there is a growing attraction for public health specialty in francophone SSA, and this trend may persist in the future. Although salary is the main motivation for the choice of public health after the love for the subject, the need for specific public health training programs will be increasing and diversifying during the next decade in francophone SSA. These are good news for the health systems of these countries because there might be more local public health specialists in the near future, with specific skills to tackle the main weaknesses of the health systems (prevention, governance, program and project management, evaluation and research) $[18,20,37,38]$. However, this could be a problem since it could lead to an increased shortage of human resources in other specialties. Therefore, it is essential for francophone SSA countries to develop a comprehensive policy framework for the management of the health workforce while taking into account all the needs of the population and promoting highshortage. This would probably require a reframing of the training system for health care professionals, and more career guidance and counseling to medical students in order to cover the gaps [19, 28, 39].

This study also revealed that about $13 \%$ of medical students wished to specialize in their home country while $58 \%$ wanted to do so abroad, mainly in Europe. It is not easy to predict whether current training capacities in public health will be able to meet the demands in francophone SSA and absorb all the students expecting to specialize in public health. In fact, there are only few master training programs in public health in francophone SSA. Except for Benin, Senegal, Mali and Burkina Faso which offer university courses leading to a master's degree in public health, the other countries only offer professional degrees or certificates 
that solely target medical graduates [16]. International initiatives such as the International Master in Applied Vaccinology involving universities from four countries (Benin, Burkina Faso, Côte d'Ivoire, and Togo), under the coordination of "Agence de Médecine Préventive" (Preventive Medicine Agency), are also being implemented to improve public health training capacities in francophone SSA. For students with very specific training needs, European and American universities offer more thorough training programs, but these often remain unaffordable without a scholarship. Distance learning courses offered by French universities are also interesting alternatives for training in public health, even if doubts remain about the ability to acquire solid skills through distance learning. This model has the advantage of avoiding expatriation (the main cause of brain drain), and is more suitable for professionals wishing to specialize in public health $[14,18,40]$. Francophone SSA universities are also developing such model of distance learning with the support of University of Geneva [21]. Increasing local training capacities is not a guarantee for the improvement of health systems of SSA countries. It would be necessary to reduce brain drain, and to recruit public health professionals in their local institutions in francophone SSA countries. Public health professionals are most often recruited by international institutions and NGOs, and are no longer available for their country of origin $[14,18,40]$.

This study was conducted using a web-based questionnaire broadcasted via student networks. This method may have introduced selection bias and members of the initial network may be overrepresented. However, this was the most effective way to reach an important number of students in different francophone SSA countries. To our knowledge, this is the first international study exploring the choice of medical specialty among pre-final and final year medical students in francophone SSA countries. The study covered 15 countries and involved more than 1,000 of medical students, providing data for the francophone part of Africa, where most of the studies have been conducted in Nigeria, Eastern and Southern Africa.

\section{Conclusion}

Public health is an emerging medical specialty in francophone SSA countries and universities are facing increasing demands for public health training. A convenient response of the academic systems in SSA is crucial, not only to meet students' demands but also to improve the health systems with a particular emphasis on preventive medicine and governance. To date, only few francophone SSA countries can meet these needs and sub-regional training initiatives must be supported so that countries may be ready to respond appropriately to future public health threats and emergencies arising in Africa.

\section{Author Statement}

\section{Contributors}

DKE conceived and designed the study and developed the initial data collection tools. DKE, BT and PAC wrote the first draft of the manuscript, which FAGK, MC, and SPE subsequently revised. DKE developed the analysis plan outline and carried out the data analyses. BT, PAC, FAGK, $\mathrm{MC}$, and SPE contributed to data analysis through review, interpretation and cross-checking figures and tables. All authors read, revised, and approved the final manuscript.

\section{Funding}

This research received no specific grant from any funding agency in the public, commercial or not-for-profit sectors.

\section{Conflict of Interest Statement}

The authors declare that they have no competing interests. Patient consent: Not required.

\section{Acknowledgements}

We are deeply indebted to the 'Réseau des Etudiants en Médecine de l'Afrique de l'Ouest" (REMAO) for their enthusiastic support.

\section{References}

[1] Epidemic focus: Lassa Fever. Releve Epidemiol Hebd. 2016 May 27; 91(21): 265-6.

[2] Nsubuga P. The Ebola outbreak in West Africa: a story of related public health challenges and a pointer to solutions to mitigate the inevitable next outbreak. Pan Afr Med J. 2014; 19: 48.

[3] Incident Management System Ebola Epidemiology Team, CDC, Guinea Interministerial Committee for Response Against the Ebola Virus; CDC Guinea Response Team, Liberia Ministry of Health and Social Welfare, CDC Liberia Response Team, Sierra Leone Ministry of Health and Sanitation, CDC Sierra Leone Response Team, et al. Update: Ebola virus disease outbreak-West Africa, October 2014. MMWR Morb Mortal Wkly Rep. 2014 Oct 31; 63(43): 978-81.

[4] Ahmed QA, Memish ZA. Yellow fever from Angola and Congo: a storm gathers. Trop Doct. 2017 Apr; 47(2): 92-6.

[5] Tarnagda Z, Cissé A, Bicaba BW, Diagbouga S, Sagna T, Ilboudo AK, et al. Dengue Fever in Burkina Faso, 2016. Emerg Infect Dis. 2018 Jan; 24(1): 170-2.

[6] World Health Organization. The World Health Report 2006 : Working together for health [Internet]. Geneva: WHO; 2006 [cited 2018 Jan 6] p. 237. (The World Health Report). Report No.: 2006. Available from: http://www.who.int/whr/2006/whr06_en.pdf?ua=1

[7] Anyangwe SCE, Mtonga C. Inequities in the Global Health Workforce: The Greatest Impediment to Health in SubSaharan Africa. Int J Environ Res Public Health. 2007 Feb; 4(2): 93-100.

[8] World Health Organization. World health statistics 2014 [Internet]. Geneva: WHO; 2014 [cited 2018 Jan 12] p. 180. Report No.: 2014. Available from: http://apps.who.int/iris/bitstream/10665/112738/1/9789240692 671_eng.pdf 
[9] GBD 2016 Mortality Collaborators. Global, regional, and national under-5 mortality, adult mortality, age-specific mortality, and life expectancy, 1970-2016: a systematic analysis for the Global Burden of Disease Study 2016. Lancet Lond Engl. 2017 Sep 16; 390(10100): 1084-150.

[10] GBD 2016 SDG Collaborators. Measuring progress and projecting attainment on the basis of past trends of the healthrelated Sustainable Development Goals in 188 countries: an analysis from the Global Burden of Disease Study 2016. Lancet Lond Engl. 2017 Sep 16; 390(10100): 1423-59.

[11] World Health Organization. Global strategy on human resources for health: Workforce 2030 [Internet]. Geneva: WHO; 2016 [cited 2018 Jan 6] p. 64. (Human Resources for Health Observer). Available from: http://apps.who.int/iris/bitstream/10665/250368/1/9789241511 131-eng.pdf

[12] World Health Organization. Health workforce requirements for universal health coverage and the sustainable development goals [Internet]. Geneva: WHO; 2016 [cited 2018 Jan 6] p. 40. (Human Resources for Health Observer). Report No.: 17. Available from:

http://apps.who.int/iris/bitstream/10665/250330/1/9789241511 407-eng.pdf

[13] Evolution and patterns of global health financing 1995-2014: development assistance for health, and government, prepaid private, and out-of-pocket health spending in 184 countries. Lancet Lond Engl. 2017 May 20; 389(10083): 1981-2004.

[14] Chen L, Evans T, Anand S, Boufford JI, Brown H, Chowdhury $\mathrm{M}$, et al. Human resources for health: overcoming the crisis. Lancet Lond Engl. 2004 Dec 27; 364(9449): 1984-90.

[15] Olafsdottir AE, Reidpath DD, Pokhrel S, Allotey P. Health systems performance in sub-Saharan Africa: governance, outcome and equity. BMC Public Health. 2011 Apr 16; 11: 237.

[16] Ijsselmuiden CB, Nchinda TC, Duale S, Tumwesigye NM, Serwadda D. Mapping Africa's advanced public health education capacity: the AfriHealth project. Bull World Health Organ. 2007 Dec; 85(12): 914-22.

[17] Kuonza L, Tint KS, Harris B, Nabukenya I. Public health systems strengthening in Africa: the role of South Africa Field Epidemiology and Laboratory Training Programme. Pan Afr Med J. 2011; 10 Supp 1: 8.

[18] Beaglehole R, Sanders D, Dal Poz M. The public health workforce in sub-Saharan Africa: challenges and opportunities. Ethn Dis. 2003; 13(2 Suppl 2): S24-30.

[19] World Health Organization. Transforming and scaling up health professionals' education and training. The world health organization guidelines 2013 [Internet]. Geneva: WHO; 2013 [cited 2018 Jan 6] p. 124. (World Health Organization 2013). Available from: http://apps.who.int/iris/bitstream/10665/93635/1/9789241506 502_eng.pdf

[20] Mokwena K, Mokgatle-Nthabu M, Madiba S, Lewis H, NtuliNgcobo B. Training of public health workforce at the National School of Public Health: meeting Africa's needs. Bull World Health Organ. 2007 Dec; 85(12): 949-54.

[21] Chastonay P, Zesiger V, Moretti R, Cremaschini M, Bailey R, Wheeler E, et al. A public health e-learning master's programme with a focus on health workforce development targeting francophone Africa: the University of Geneva experience. Hum Resour Health. 2015 Aug 13; 13: 68.

[22] Ballew P, Castro S, Claus J, Kittur N, Brennan L, Brownson RC. Developing web-based training for public health practitioners: what can we learn from a review of five disciplines? Health Educ Res. 2013 Apr; 28(2): 276-87.

[23] Celletti F, Reynolds TA, Wright A, Stoertz A, Dayrit M. Educating a new generation of doctors to improve the health of populations in low- and middle-income countries. PLoS Med. 2011 Oct; 8(10):e1001108.

[24] Frenk J, Chen L, Bhutta ZA, Cohen J, Crisp N, Evans T, et al. Health professionals for a new century: transforming education to strengthen health systems in an interdependent world. Lancet Lond Engl. 2010 Dec 4; 376(9756): 1923-58.

[25] Diallo I. [Public health training in subsaharan Africa: challenges and opportunities]. Med Trop Rev Corps Sante Colon. 2004; 64(6): 595-602.

[26] Rabbani F, Shipton L, White F, Nuwayhid I, London L, Ghaffar A, et al. Schools of public health in low and middleincome countries: an imperative investment for improving the health of populations? BMC Public Health. 2016 Sep 7; 16: 941.

[27] Dossajee H, Obonyo N, Ahmed SM. Career preferences of final year medical students at a medical school in Kenya--A cross sectional study. BMC Med Educ. 2016 Jan 11; 16: 5.

[28] Ossai EN, Uwakwe KA, Anyanwagu UC, Ibiok NC, Azuogu BN, Ekeke N. Specialty preferences among final year medical students in medical schools of southeast Nigeria: need for career guidance. BMC Med Educ [Internet]. 2016 Oct 4; 16. Available from:

https://www.ncbi.nlm.nih.gov/pmc/articles/PMC5050581/

[29] Egbi OG, Unuigbe EI. Choice of medical specialties amongst final year medical students in two universities in South-South, Nigeria. West Afr J Med. 2014 Mar; 33(1): 44-50.

[30] Gorenflo DW, Ruffin MT, Sheets KJ. A multivariate model for specialty preference by medical students. J Fam Pract. 1994 Dec; 39(6): 570-6.

[31] Lefevre JH, Roupret M, Kerneis S, Karila L. Career choices of medical students: a national survey of 1780 students. Med Educ. 2010 Jun 1; 44(6): 603-12.

[32] Abdulghani HM, Al-Shaikh G, Alhujayri AK, Alohaideb NS, Alsaeed HA, Alshohayeb IS, et al. What determines the selection of undergraduate medical students to the specialty of their future careers? Med Teach. 2013; 35 Suppl 1:S25-30.

[33] Muula AS, Komolafe OO. Specialisation patterns of medical graduates, University of Malawi College of Medicine, Blantyre. Cent Afr J Med. 2002 Feb; 48(1-2): 14-7.

[34] Aluttis C, Bishaw T, Frank MW. The workforce for health in a globalized context-global shortages and international migration. Glob Health Action [Internet]. 2014 Feb 13 [cited 2018 Jan 6]; 7. Available from: https:/www.ncbi.nlm.nih.gov/pmc/articles/PMC3926986/

[35] World Health Organization. Framing the health workforce agenda for the Sustainable Development Goals. Geneva: World Health Organization; 2017 p. 44. Report No.: WHO/HIS/HWF/biennium report/2017.1. 
[36] Omoleke SA, Mohammed I, Saidu Y. Ebola Viral Disease in West Africa: A Threat to Global Health, Economy and Political Stability. J Public Health Afr [Internet]. 2016 Aug 17 [cited 2018 Jan 12]; 7(1). Available from: https://www.ncbi.nlm.nih.gov/pmc/articles/PMC5349256/

[37] McKee M. Seven goals for public health training in the 21st century. Eur J Public Health. 2013 Apr; 23(2): 186-7.

[38] Rabbani F, Shipton L, White F, Nuwayhid I, London L, Ghaffar A, et al. Schools of public health in low and middle- income countries: an imperative investment for improving the health of populations? BMC Public Health. 2016 Sep 7; 16: 941.

[39] Watts G. Tolullah Oni: public health specialist with a global ambition. Lancet Lond Engl. 2016 Apr 23; 387(10029): 1712.

[40] Narasimhan V, Brown H, Pablos-Mendez A, Adams O, Dussault G, Elzinga G, et al. Responding to the global human resources crisis. Lancet Lond Engl. 2004 May 1; 363(9419): 1469-72. 